\title{
Adaptação do questionário internacional de atividade física para idosos
}

\author{
Adaptation of the international physical activity questionarie \\ for the elderly
}

\begin{abstract}
1 Universidade do Estado de Santa Catarina. Centro de Ciências da Saúde e do Esporte. Laboratório de Gerontologia. Florianópolis, SC. Brasil.
\end{abstract}

2 Universidade Federal de Santa Catarina. Programa de Pós-Graduação em Educação Física. Florianópolis, SC.

Recebido em 03/02/10 Revisado em 15/04/10 Aprovado em 01/06/10
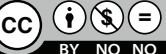

Resumo - O Questionário Internacional de Atividade Física - IPAQ estima o dispêndio energético semanal de atividades físicas (AF). Ele foi validado para idosos brasileiros. Diante de dificuldades apresentadas pelos idosos quanto à mensuração da quantidade de dias, do tempo e da intensidade na realização das AF (domínios), durante uma semana normal, e pelos entrevistadores na pesquisa de campo, surgiu a necessidade de adaptação na estrutura e aplicação do IPAQ. O IPAQ adaptado é composto por 5 domínios e 15 questões; é aplicado em forma de entrevista, devido ao baixo nível de escolaridade dos idosos; no modelo longo, pela melhor discriminação nas AF em cada domínio; referente a uma semana normal, auxiliando no recordatório durante os turnos (manhã, tarde e noite); e com detalhamento sobre a intensidade leve, moderada e vigorosa. $O$ treinamento dos entrevistadores é fundamental. O relatório do IPAQ adaptado entregue ao idoso deve ser em minutos por semana e usar a classificação de "ativos" (>= $150 \mathrm{~min} / \mathrm{sem}$ ). O IPAQ adaptado para idosos é um instrumento internacional, com validade para a população idosa brasileira, de fácil aplicação, baixo custo financeiro, que atinge grandes grupos populacionais, método não invasivo, entre outras vantagens.

Palavras-chave: Questionário; Adaptação; Atividade Física; Idosos.

Abstract - The International Physical Activity Questionnaire (IPAQ) estimates the weekly energy expenditure for physical activities (PA). This instrument was validated for use with Brazilian older adults. The need to adapt the structure and application of the IPAQ arose from the difficulties of older adults to correctly measure the number of days, time and intensity of PA (domains) during a normal week and the difficulties encountered by the interviewers in field research. The adapted IPAQ consists of five domains and 15 questions and is applied by interview because of the low educational level of Brazilian older adults. The instrument is applied in its long form to permit better discrimination of PA in each domain and refers to a normal week to help recall morning, afternoon and evening activities. In addition, it includes details about mild, moderate and vigorous intensity. The training of the interviewers is fundamental. The report of the IPAQ adapted for older adults should be delivered in minutes per week and should use the classification active ( $\leq 150 \mathrm{~min} /$ week). The IPAQ adapted for older adults is an international instrument that is valid for the Brazilian elderly population. The instrument is a noninvasive easily applied method of low cost that reaches large population groups, among other advantages. Key words: Questionnaire; Adaptation; Physical activity; Older adults. 


\section{INTRODUÇÃO}

Existem alguns questionários utilizados para mensurar o nível de atividade física em idosos, dentre eles, destaca-se o Questionário Internacional de Atividade Física- IPAQ que é um instrumento que permite estimar o dispêndio energético semanal de atividades físicas relacionadas com o trabalho, transporte, tarefas domésticas e lazer, realizadas por, pelo menos, 10 minutos contínuos, com intensidade moderada e vigorosa, durante uma semana normal/ habitual. O IPAQ apresenta as formas longa e curta e pode ser aplicado pelo telefone ou ser autoadministrado, tanto como recordatório dos últimos 7 dias quanto de uma semana normal/habitual ${ }^{1,2}$.

O IPAQ foi desenvolvido a partir das dificuldades encontradas para se obter medidas de atividades físicas internacionalmente comparáveis. A Organização Mundial de Saúde, o Centro de Controle e Prevenção de Doenças dos Estados Unidos e o Instituto Karolinska, na Suécia, reuniram os pesquisadores da área de atividade física e saúde para desenvolver e testar um instrumento de medida da atividade física para uso internacional. Em 2001, o Comitê Executivo responsável por desenvolver o IPAQ publicou um relatório parcial desta aplicação experimental ${ }^{1,2}$.

No Brasil, o estudo de validade e reprodutibilidade do IPAQ, em adultos, foi coordenado pelo Centro de Estudos do Laboratório de Aptidão Física de São Caetano do Sul, em São Paulo, tendo sido aplicado em adultos. Os resultados foram similares ao internacional e a outros instrumentos que mensuram o nível de atividade física ${ }^{3}$.

Paralelamente a esse estudo, outros foram conduzidos no Brasil ${ }^{4-10}$, visando avaliar as versões correntes do IPAQ quanto à reprodutibilidade (teste-reteste) e validade (face e concorrente). Em geral, os resultados encontrados nesses estudos demonstraram que o IPAQ é um instrumento com boa estabilidade de medidas e precisão aceitável para uso em estudos populacionais com adultos jovens e de meia idade.

Os estudos apontados acima avaliaram as versões concorrentes do IPAQ para adultos jovens e de meia idade brasileiros. Em relação as pessoas idosas, foram realizados estudos ${ }^{11,12}$ com o objetivo de verificar a reprodutibilidade e validade do IPAQ (forma longa e semana normal/habitual) com mulheres e homens idosos. Os resultados do IPAQ para os idosos de ambos os sexos apresentaram um nível de validade concorrente moderado, sendo mais indicado para discriminar níveis gerais de AF (menos ativos/mais ativos).
Quanto à aplicação do IPAQ em população idosa $^{13,14}$, algumas dificuldades foram apresentadas pelos idosos quanto à mensuração da quantidade de dias (semana normal/habitual), do tempo (horas e minutos por dia e semana) e da intensidade (leve ou moderada ou vigorosa) na realização das atividades físicas nos diferentes domínio do IPAQ (trabalho, transporte, tarefas domésticas, lazer e tempo sentado). Há necessidade de um treinamento específico com os coletadores de dados devido às características específicas dos idosos.

Diante dessas dificuldades, surge a necessidade de adaptar o IPAQ quanto à sua estrutura e aplicação, sem alterar a ordem e a linguagem das questões, para não afetar as propriedades psicométricas do instrumento.

Conforme Bueno ${ }^{15}$, adaptar significa ajustar uma coisa a outra, moldar e apropriar. Assim, a adaptação do IPAQ para idosos faz-se necessária pelas dificuldades apresentadas pelos idosos e pelos entrevistadores, bem como, pela experiência científica, acadêmica e de intervenção das autoras na aplicação do instrumento em idosos, em suas teses de doutorado ${ }^{13,14}$

Diante disto, o presente estudo teve como objetivo propor adaptações na estrutura e aplicação no IPAQ, para idosos.

\section{ESTRUTURA DO IPAQ ADAPTADO PARA IDOSOS}

O IPAQ (forma longa e semana normal/habitual ou dos últimos 7 dias) original é composto por 5 domínios e 27 questões e o IPAQ adaptado para idosos é composto pelos mesmos domínios, mas apresenta um número menor de questões (15), porque foram agrupadas na mesma questão as perguntas referentes aos "dias da semana" e ao "tempo (min/horas dia)" de determinada atividade física, conforme mostra o quadro 1 .

O IPAQ adaptado para idosos e seus domínios, número de questões e objetivos estão descrito abaixo:

Domínio 1 - Atividades físicas no trabalho Este domínio, composto de 4 questões, tem como objetivo verificar o nível de atividade física durante o trabalho, remunerado ou voluntário, que os idosos realizam em uma semana normal/habitual e a intensidade das atividades físicas (moderada ou vigorosa), durante o mínimo de 10 minutos contínuos. 
Quadro 1- Domínios e número de questões do IPAQ original e do adaptado para idosos.

\begin{tabular}{lcc}
\hline Domínios do IPAQ & $\begin{array}{c}\text { № questões } \\
\text { original }\end{array}$ & $\begin{array}{c}\text { No questões adaptado } \\
\text { 1- Atividades físicas no trabalho }\end{array}$ \\
2- Atividades físicas como meio de transporte & 06 & 03 \\
3- Atividades físicas em casa: tarefas domésticas e família & 06 & 03 \\
4- Atividades físicas de recreação, esporte exercício físico e de lazer & 06 & 03 \\
5- Tempo gasto sentado & 02 & 02 \\
\hline
\end{tabular}

Domínio 2 - Atividades físicas como meio de transporte Este domínio, composto de três questões, tem como objetivo verificar como os idosos se deslocam de um lugar para outro, em uma semana normal/ habitual, incluindo todos os meios de transporte (ônibus, carro, moto, bicicleta...) e a pé (não incluir as caminhadas de lazer e como exercício físico). São consideradas as atividades físicas com duração de, pelo menos, 10 minutos contínuos e sua intensidade (moderada ou vigorosa).

Domínio 3 - Atividades físicas em casa: tarefas domésticas e família

Este domínio, composto de três questões, tem como objetivo verificar as atividades físicas que o idoso realiza em uma semana normal/habitual dentro e ao redor da sua habitação (casa ou apartamento). São consideradas as atividades físicas com duração de, pelo menos, 10 minutos contínuos e sua intensidade (moderada ou vigorosa).

Domínio 4 - Atividades físicas de recreação, esporte, exercício físico e de lazer

Este domínio, composto de três questões, refere-se às atividades físicas que o idoso faz em uma semana normal/habitual de atividades físicas de recreação, esporte, exercício físico e lazer. São consideradas as atividades físicas com duração de, pelo menos, 10 minutos contínuos e sua intensidade (moderada ou vigorosa).

Domínio 5 - Tempo gasto sentado

Este domínio, composto de duas questões, refere-se ao tempo em que o idoso permanece sentado em casa, no grupo de convivência de idoso, na visita a amigos e parentes, na igreja, no consultório médico, fazendo trabalhos manuais e no trabalho, durante seu tempo livre. Não é incluído o tempo gasto sentado durante o transporte em ônibus e/ou carro. É computado o tempo (minutos e horas) sentado durante um dia de uma semana normal/habitual e em um dia do final de semana.

$\mathrm{Na}$ adaptação do IPAQ para idosos também foram modificados alguns exemplos que eram apre- sentados no instrumento original, com intuito de facilitar as repostas dos idosos a partir de exemplos relacionados com o seu contexto social e cultural. Como exemplo, no domínio 2 - Atividades físicas como meio de transporte, no deslocamento a pé (caminhando), colocaram-se exemplos como ir à igreja, ao médico e ao grupo de convivência, atividades estas que fazem parte da rotina da maioria dos idosos.

O modelo do IPAQ adaptado para idosos encontra-se disponível nos seguintes estudos: Benedetti et al..$^{12}$, Mazo ${ }^{13}$ e Benedetti ${ }^{14}$.

\section{RECOMENDAÇÕES PARA A APLICAÇÃO DO IPAQ ADAPTADO PARA IDOSOS}

A aplicação do IPAQ longo adaptado para idosos deve seguir as recomendações, abaixo:

a) Com o idoso

- Em estudos com a população idosa, a entrevista frente a frente é o melhor procedimento para a aplicação de questionários e deve ser realizado em local silencioso.

b) $\mathrm{O}$ entrevistador:

- Realizar as etapas da entrevista, seguindo as normas e padrões éticos, desde o momento para a prepração para a entrevista, contato com os idosos, a entrevista propriamente dita e o encerramento desta;

- Comunicar ao Supervisor da pesquisa a ocorrência de problema durante a operação de campo, que possa trazer danos a qualidade da pesquisa;

- Preservar o material de trabalho, tais como questionários, pranchetas, canetas entre outros.

c) Treinamento dos entrevistadores:

- A capacitação adequada dos entrevistadores é fundamental para o sucesso de uma pesquisa. Os entrevistadores devem estar preparados para desenvolver suas tarefas. $\mathrm{O}$ treinamento dos entrevistadores é importante para homogeneizar a forma de aplicação do IPAQ adaptado para idosos. 
d) Aplicação do IPAQ adaptado para idosos:

- Utilizar a forma longa do IPAQ para idosos, pois esta consegue discriminar melhor as atividades físicas em cada domínio, ajudando no recordatório das atividades realizadas pelos idosos numa semana normal/habitual;

- No IPAQ adaptado, foram agrupadas, na mesma questão, as perguntas referentes "aos dias" e "ao tempo" dos diferentes domínios da atividade física (quais os dias e qual o tempo numa semana normal e por 10 minutos contínuos). Para resposta, foi construído um quadro com os dias da semana e turnos. No quadro, o entrevistador deverá anotar o tempo (minutos e horas) gasto em atividade física moderada e/ ou vigorosa em cada dia da semana e turnos (manhã, tarde e noite). No final da entrevista, o entrevistador deverá somar os tempos (minutos e horas/dia) e anotar em cada questão o valor total em minutos e horas e dos dias da semana $^{12-14}$.

- O entrevistador deverá questionar sobre todas as atividades físicas realizadas pelo idoso nos diferentes domínios do IPAQ, quanto ao tempo (em minutos e horas por dia), fazendo com que ele recorde o que fez durante os turnos (manhã, tarde e noite) nos dias de sua semana normal/habitual. No recordatório das atividades durante o dia, o idoso deve relatar sobre as atividades realizadas desde o acordar até o dormir. Durante este relato, o entrevistador deve questionar e anotar o tempo (minutos e horas) dispendido com estas atividades e a sua intensidade (moderada ou vigorosa).

- Deve-se prestar atenção em algumas dificuldades que os idosos apresentam, como de diferenciar as atividades de intensidade leve, moderada e vigorosa. Como exemplo, os idosos, geralmente, colocam que lavar a louça, estender a roupa são atividades moderadas, mas são classificadas como leve. Diante disto, em cada pergunta deve-se reforçar o que é atividade leve, moderada e vigorosa, conforme a orientação do instrumento ${ }^{12-14}$

- Deve-se ter atenção quanto ao questionamento sobre o tempo sentado numa semana normal e um dia do final de semana, pois muitas vezes, os idosos relatam que não ficam muito tempo sentados durante o dia, pois desconsideram o tempo sentado durante as refeições, fazendo trabalhos manuais e o tempo sentado assistindo televisão. Então a pergunta tem que detalhar os exemplos do instrumento.

\section{TRATAMENTO DOS DADOS DO IPAQ}

A equipe que criou o IPAQ recomenda a análise em METs, mas como pesquisadores sugerimos que em pesquisa também seja utilizada minutos/semana e/ou Kcal. O resultado do IPAQ em minutos/ semana e sua classificação possibilitam uma melhor visualização e entendimento do idoso sobre o seu nível de atividade física.

Assim, recomenda-se que no relatório entregue ao idoso seja apresentado o resultado do IPAQ em minutos por semana, por considerar que este é um critério baseado em recomendações atuais de limiares de atividades físicas que resultam em benefícios para a saúde, classificando como "ativos" os indivíduos que praticam, pelo menos, 150 minutos por semana ( $\mathrm{min} / \mathrm{sem}$ ) de atividade física, no mínimo moderada ${ }^{1}$.

\section{CONSIDERAÇÕES FINAIS}

Diante do aumento do contingente da população idosa brasileira que, muitas vezes, é acompanhado pelo sedentarismo, doenças crônico-degenerativas, dependência e incapacidades, a avaliação do nível de atividade física dos idosos é recomendada, pois, por meio desta, pode-se propor intervenções e orientações de práticas de atividades físicas adequadas quanto à quantidade, intensidade e frequência, que busquem manter ou melhorar o nível de atividade física e a capacidade funcional dos idosos, atingindo critérios de recomendáveis.

Assim, o Questionário Internacional de Atividade Física- IPAQ (forma longa, semana normal/ habitual e aplicado com entrevista) validado para idosos brasileiros por Benedetti et al. ${ }^{11}$ e Benedetti et al. ${ }^{12}$ e adaptado pelas autoras deste ponto de vista, é uma boa opção para avaliar o nível de atividade física dos idosos, pois é um instrumento aplicado internacionalmente, com validade para a população idosa brasileira, de fácil aplicação, baixo custo financeiro, permite atingir grandes grupos populacionais, é um método não invasivo, de baixo risco ao indivíduo, entre outras vantagens. $\mathrm{O}$ instrumento está disponível nas seguintes referências instrumento ${ }^{12-14}$.

\section{REFERÊNCIAS BIBLIOGRÁFICAS}

1. Marshall A, Bauman A. The International Physical Activity Questionnaire. Summary Report of the Reliability \& Validity Studies. Produzido pelo Comitê Executivo do IPAQ. Summary, March, 2001. 
2. Craig, CL, Marshall AL, Sjostrom M, Bauman A E, Booth Ml, Ainsworth Be, Pratt M, Ekelund ULF, Yngve A, Sallis JF, Oja P. International Physical Activity Questionnaire: 12-Country Reliability and Validity. Med Sci Sports Exerc 2003;35(8):1381-95.

3. Matsudo S, Araújo T, Matsudo V, Andrade D, Andrade E, Oliveira C, Braggion G. Questionário Internacional de Atividade Física (IPAQ): estudo de validade e reprodutibilidade no Brasil. Rev Bras Ativ Fís Saúde 2001; 6(2):5-12.

4. Pardini R, Matsudo S, Matsudo V, Araújo T, Andrade E, Braggion G, Andrade D, Oliveira L, Figueira Junior A, Raso V. Validation os International Physical Questionnaire (IPAQ): pilot study in Brazilian Young adults. Med Sci Sports Exerc 1997;29(6):S5-S9.

5. Barros M, Nahas M. Reprodutibilidade (teste/reteste) do Questionário Internacional de Atividade Física (QIAF-versão 6): um estudo piloto com adultos no Brasil. Rev Bras Ciên Mov 2000;8(1):23-6.

6. Silva DK, Rebelo, FPV, Ramos JH, Barros MVG, Nahas M. Reprodutibilidade e validade concorrente do Questionário Internacional de Atividade Física (QIAF-versão 8). (Resumo). Anais do $8^{\circ}$ Congresso de Educação Física e Ciências do Esporte dos Países de Língua Portuguesa. 2000; p. 382.

7. Silva DK, Rojas PNC, Teive, PF, Barros MVG, Nahas M. Reprodutibilidade do Questionário Internacional de Atividade Física (IPAQ- 8). (Resumo). Anais do XXIV Simpósio Internacional de Ciências do Esporte. 2001; p. 139.

8. Hallal PC, Victora CG, Lima RC. Validando atividade física: estudo de validação da versão curta do IPAQ (Resumo). Rev Bras Epidemiol. Anais do V Congresso Brasileiro de Epidemiologia; 2002.

9. Hallal PC, Victora CG Reliability and validity of the international physical activity questionnaire (IPAQ) (Abstract). Med Sci Sports Exerc 2004;36(3):556.

10. Guedes DP, Lopes CC, Guedes Jerp. Reprodutibilidade e validade do Questionário Internacional de Atividade Física em adolescentes. Rev Bras Med Esporte 2005;11(2):151-8
11. Benedetti TRB, Mazo GZ, Barros MVG. Aplicação do Questionário Internacional de Atividade Físicas para avaliação do nível de atividades físicas de mulheres idosas: validade concorrente e reprodutibilidade teste/ reteste. Rev Bras Ciên Mov 2004;12(1):25-33.

12. Benedetti TRB, Antunes PC, Rodriguez-Añez CR, Mazo GZ, Petroski EL. Reprodutibilidade e validade do questionário internacional de atividade física (IPAQ) em homens idosos. Rev Bras Med Esporte 2007;13(1):11-6.

13. Mazo GZ. Atividade física e qualidade de vida de mulheres idosas. Faculdade de Ciências de Desportos e de Educação Física da Universidade do Porto. [Tese de doutorado] Porto - Portugal, 2003.

14. Benedetti TRB. Atividade física: uma perspectiva de promoção de saúde do idoso no município de Florianópolis. Florianópolis [Tese de Doutorado]. Florianópolis; 2004.

15. Bueno FS. Minidicionário da Lingua Portuguesa. São Paulo: Lisa S.A, 1998.

\section{Endereço para correspondência}

Giovana Zarpellon Mazo

Endereço: Rua Paschoal Simone, 358

Coqueiros.

CEP: 88080-350 - Florianópolis / SC

E-mail: d2gzm@udesc.br benedetti@cds.ufsc.br 